\title{
Impact of Subclinical Rejection on Kidney Graft Function During the First Year after Transplantation
}

\author{
Vadims Suhorukovs, Janis Jushinskis, Rafail Rozental \\ Latvian Transplantation Center, Pauls Stradins Clinical University Hospital, Riga, Latvia
}

\section{Summary}

Introduction. There is no unified opinion about the role of subclinical rejection (SR) in further kidney graft function and the necessity of its treatment.

Aim of the Study. The aim of our study is to determine the impact of SR on kidney graft function during the first year after transplantation.

Materials and methods. Totally 144 deceased donor kidney transplantations were performed in a single center between January 1 , 2007 , and March 16, 2009. The study included patients who developed the primary graft function $(n=78)$. Protocol biopsies were performed in 28 patients. The patients were divided into 4 groups: group A - patients, who had no histological signs of rejection on their protocol biopsy; group B - patients with histological signs of rejection who were treated with steroids; group C - patients with histological signs of rejection and with no treatment; group D - patients who were not biopsied.

All groups were compared for serum creatinine level, glomerular filtration rate (GFR), number of clinical rejection 1, 3, 6, 12 months after the transplantation.

Results. Histological examination revealed that 18 of 28 patients who underwent the protocol biopsy had SR grade from IA to IIA (64.3\%). Comparison of the group showed no statistical difference in creatinine level and GFR 12 months after the transplantation ( $p>0,05$ for all groups).

Conclusions. During the first 12 months after transplantation SR does not significantly impact the graft function. However, the graft function was slightly worse in patients with SR and without treatment, and relatively better in patients with SR who were treated by steroids than in patients from others groups. Further follow-up is needed to determine the longer-term results.

Key words: kidney transplantation, subclinical rejection, protocol biopsy.

\section{INTRODUCTION}

Recently, there has been a considerable improvement of kidney transplantation results. During the last decade, the number of acute transplant rejection cases and transplant loss within early post transplant period has significantly reduced. But despite the good shortterm results the adequate improvement of long-term results has not been achieved and the time limited renal graft function remains the serious problem of current transplantology. Meier-Kriesche et al. reported that relative risk for overall renal transplant loss in 1996 was 1.0, but in 2000 it was 1.14 (2).

The term of subclinical rejection (SR) was introduced during the nineties to denote histological signs of acute rejection in kidney graft with stable function (8). Some studies have established association between SR and chronic allograft nephropathy (CAN) development (4). However, there still exists no unified opinion about the role of subclinical rejection in further kidney graft function (3) and the necessity of its treatment, and protocol biopsy is still not standard of care at many transplant centers (5).

\section{AIM OF THE STUDY}

The aim of this study was to determine the impact of SR on kidney graft function during the first year after transplantation and to clarify the effect of its treatment.

\section{MATERIALS AND METHODS}

Totally 144 deceased donor kidney transplantations were performed in a single center between January 1, 2007, and March 16, 2009. The study included 78 patients who expressed their informed consent and who developed primary graft function and without any complications during 1 month after transplantation acute clinical rejection, delayed graft function, severe infection, urinoma, post operation hematoma that needed reoperation etc. Immunosuppression included induction by monoclonal (basilixumab or daclizumab) or polyclonal (ATG) antibodies with a 5-day steroid pulse, and maintenance by per oral steroids, mycophenolate mofetil and Neoral as guided by the blood level - about $150 \mathrm{ng} / \mathrm{ml}$ during the first month after transplantation. Protocol biopsies - the planned biopsy without direct indications - were performed on 28 patients who gave their agreement and who met the following criteria during 10 days before the biopsy: serum creatinine $<0.2$ $\mathrm{mmol} / \mathrm{l}$, adequate diuresis, normal body temperature and stable renal graft function defined as variability of serum creatinine of less than $20 \%$. Protocol biopsies were performed within the 3rd and 4th weeks after transplantation under ultrasound guidance. Histological changes were diagnosed according to the 1997 Banff criteria $^{7}$. Subclinical rejection was defined as the presence of histological changes grade from IA.

All patients were divided into 4 groups: group A - 
patients, who had no histological signs of rejection on their protocol biopsy; group B - patients with histological signs of rejection who were treated by steroid $(500 \mathrm{mg}$ i/v for 3 days); group $\mathrm{C}$ - patients with histological signs of rejection and with no treatment; group D - patients who underwent no protocol biopsy.

All groups were compared for age and gender of the recipient, age of the donor, cold ischemia time, serum creatinine level and GFR (by Cockcroft-Gault equation) $1,3,6$ and 12 months after transplantation and number of acute clinical rejection and graft loss during the first year after transplantation.

Descriptive statistics were used to summarize the demographic and clinical features. Results were expressed as mean \pm SD. One-way ANOVA analysis was used to compare groups for parametric variables, and Pearson's Chi-squared test - for non-parametric variables.

Only $\mathrm{p}<0,05$ was considered statistically significant. All statistical analyses were performed using SPSS 13,0 (SPSS Inc.).

\section{RESULTS}

Histological examination revealed that 18 of 28 patients who had undergone protocol biopsy had SR grade from IA to IIA $(64.3 \%)$ : grade IA had 13 patients; IB 4 patients; IIA - 1 patient (Figure 1). Comparison of the groups showed no statistically significant difference ( $p>0,05$ between all groups) in age and gender of the recipient, age of the donor, cold ischemia time, acute clinical rejection number and graft loss number during 12 month after transplantation (Table 1). One patient from group B has lost his graft due to non-compliance of immunosuppression therapy.

Comparison of the groups showed statistically significant difference in serum creatinine level at 1 month after transplantation between the group B and group D $(\mathrm{p}=$ $0,043)$ and between the group $\mathrm{C}$ and group $\mathrm{D}(\mathrm{p}=$ $0,027)$; but at 3,6 , and 12 months after transplantation this difference became not statistically significant $(p>$ $0,05)$. The differences in serum creatinine level and GFR at 1, 3, 6, 12 months after the transplantation between other groups had no statistical significance $(p>0,05)$. However, group $\mathrm{C}$ patients at 12 months had relatively higher mean serum creatinine level and lower GFR compared with other group patients, but group B patients, although statistically insignificant, at 12 months had the mean serum creatinine level slightly lower and GFR slightly higher than in patients from others groups.

\section{DISCUSSION}

Most sources currently acknowledge the presence of subclinical rejections. According to the data from various authors, their numbers in early posttransplant period lies between 7 and $46.6 \%(3,4,9)$. High SR rate in our study could be explained by a small number of patients who underwent the protocol biopsy and also probably by specificities of immunosuppressive therapy used in our center (rapid decrease of the steroid dose and relatively low doses of Cyclosporine) and high rate of HLA mismatches in our patients.

However, there is no common opinion on the role of SR and on the necessity of its treatment. Some authors note that with time SR could progress into an acute clinical rejection or persist subclinically, facilitating development of chronic transplant nephropathy resulting in graft malfunction $(1,4,6)$. Our study showed that in the first 12 months following the transplantation the number of acute clinical rejections in patients with no signs of SR and those who got steroid treatment because of SR, does not differ from the number of acute clinical rejections in patients with signs of SR who got no treatment. As for the transplant functioning, 12 months after the operation the patients with no treatment revealed deterioration, although statistically insignificant, of the function compared to other groups of patients.

Group D comprised patients who had no protocol biopsies (mostly due to refusals by the patients). However, proceeding from the data we obtained, it could be assumed that this group also includes patients with signs of SR that remained untreated. This confirms the fact that after 12 months the creatinine level in this group is higher, although statistically insignificantly, than in Groups A and B.

At the same time, Group B patients who got steroid treatment, 12 months after reveal, although statistically insignificantly, a lower creatinine level and a higher GFR level compared to other groups.

\section{CONCLUSIONS}

Results of our study show that during the first 12 months after transplantation SR does not significantly impact the kidney graft function. However, the graft function was slightly worse in patients with SR and without treatment (group C) and relatively better in patients with SR who were treated by steroids (group B) than in patients from others groups and further followup is needed to determine the longer-term results.

\section{Conflict of interest: None}

\section{REFERENCES}

1. Kumar MSA, Heifets M, Moritz MJ et al. Safety and efficacy of steroid withdrawal two days after kidney transplantation: analysis of results at three years. Transplantation 2006;81:832-839

2. Meier-Kriesche H-U, Schold JD, Srinivas TR et al. Lack of improvement in renal allograft survival despite a marked decrease in acute rejection rates over the most recent era. Am J Transplant 2004; 4:378-383

3. Moreso F., Ibernon M., Goma M. et al. Subclinical rejection associated with chronic allograft nephropathy in protocol biopsies as a risk factor for late graft loss. Am J Transplant 2006; 6:747-752

4. Moriatsu Miyagi, Yukio Ishikawa, Sonoo Mizuiri et al. Significance of subclinical rejection in early renal allograft biopsies for chronic allograft dysfunction. Clin Transplant 2005; 19:456- 465 
5. Nankivell BJ, Chapman JR. The significance of subclinical rejection and the value of protocol biopsies. Am J Transplant 2006; 6:2006-2012

6. Nankivell BJ, Borrows RJ, Fungs CLS et al. Natural history, risks factors and impact of subclinical rejection in kidney transplantation. Transplantation 2004; 78:242-249

7. Racusen LC, Solez K, Colvin RB et al. The Banff 97 working classification of renal allograft pathology. Kidney Int 1999; 55:713-723

8. Rush DN, Jeffery JR, Gough J. Sequential protocol biopsies in renal transplant patients. Transplantation 1995; 59:511-514

9. Terence Y-S Kee, Chapman JR, O'Connell PJ et al. Treatment of subclinical rejection diagnosed by protocol biopsy of kidney transplants. Transplantation 2006; 82:36-42

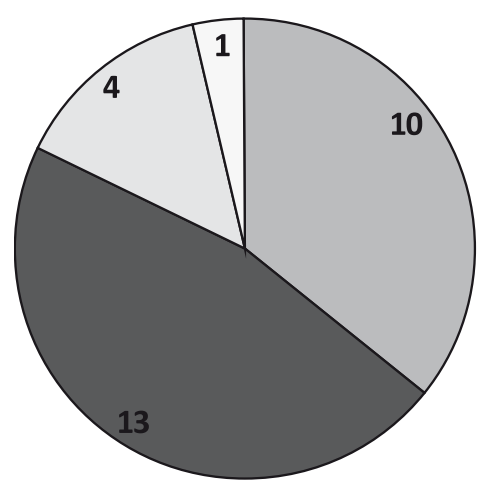

$\square$ no rejection
$\square$ grade IA
$\square$ grade IB
$\square$ grade IIA

Fig. 1. Number of subclinical rejection cases

Table 1. Demographic and clinical features of the groups

\begin{tabular}{|c|c|c|c|c|}
\hline Variables & $\begin{array}{c}\text { Group A } \\
(\mathrm{n}=10)\end{array}$ & $\begin{array}{l}\text { Group B } \\
(\mathrm{n}=10)\end{array}$ & $\begin{array}{l}\text { Group } C \\
(\mathrm{n}=8)\end{array}$ & $\begin{array}{l}\text { Group D } \\
(\mathrm{n}=50)\end{array}$ \\
\hline Donor age $(y r)$ mean \pm SD & $41.4 \pm 10.1$ & $52.9 \pm 13.4$ & $48.6 \pm 8.4$ & $42.4 \pm 14.7$ \\
\hline Recipient age $(y r)$ mean $\pm S D$ & $50.5 \pm 11.4$ & $47.8 \pm 15.4$ & $49 \pm 7.5$ & $46.4 \pm 15.1$ \\
\hline Recipient gender $(\mathrm{M} / \mathrm{F})$ & $5 / 5$ & $5 / 5$ & $3 / 5$ & $18 / 32$ \\
\hline Cold ischemia time $(\mathrm{h})$ mean $\pm \mathrm{SD}$ & $16.9 \pm 2.9$ & $16.9 \pm 1.9$ & $16.3 \pm 2.8$ & $15.7 \pm 14.7$ \\
\hline $\begin{array}{l}\text { Clinical acute rejection during l year, } \\
\text { number of cases }\end{array}$ & 3 & 2 & 2 & 6 \\
\hline Graft loss during 1 year, number of cases & 0 & 1 & 0 & 2 \\
\hline $\begin{array}{l}\text { Serum creatinine }(\mathrm{mmol} / \mathrm{l}) \text { mean } \pm \text { SD } \\
1 \text { month } \\
3 \text { months } \\
6 \text { months } \\
12 \text { months }\end{array}$ & $\begin{array}{l}0.112 \pm 0.021 \\
0.116 \pm 0.025 \\
0.122 \pm 0.027 \\
0.131 \pm 0.029\end{array}$ & $\begin{array}{l}0.121 \pm 0.027 \\
0.121 \pm 0.027 \\
0.128 \pm 0.023 \\
0.128 \pm 0.023\end{array}$ & $\begin{array}{l}0.125 \pm 0.035 \\
0.131 \pm 0.037 \\
0.138 \pm 0.044 \\
0.150 \pm 0.054\end{array}$ & $\begin{array}{l}0.106 \pm 0.019 \\
0.113 \pm 0.023 \\
0.122 \pm 0.031 \\
0.135 \pm 0.031\end{array}$ \\
\hline 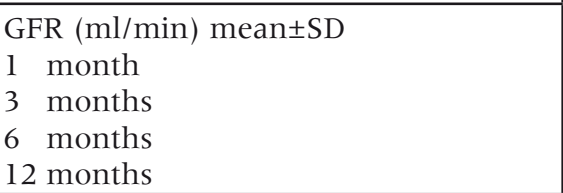 & $\begin{array}{l}72.3 \pm 15.9 \\
70.3 \pm 16.0 \\
66.4 \pm 13.0 \\
59.8 \pm 15.9\end{array}$ & $\begin{array}{l}68.4 \pm 21.9 \\
68.4 \pm 21.9 \\
63.2 \pm 21.0 \\
64.0 \pm 20.7\end{array}$ & $\begin{array}{l}59.8 \pm 11.6 \\
57.1 \pm 12.3 \\
55.4 \pm 12.8 \\
52.4 \pm 14.5\end{array}$ & $\begin{array}{l}74.5 \pm 16.7 \\
70.7 \pm 17.6 \\
67.2 \pm 18.4 \\
61.2 \pm 17.0\end{array}$ \\
\hline
\end{tabular}

\section{Address:}

Vadims Suhorukovs

Pauls Stradins Clinical University Hospital,

13 Pilsonu Street, Riga,

LV-1002, Latvia;

E-mail: vadim.suhorukov@inbox.lv 